\title{
Gold Labeling of Protein Fusion Tags for EM
}

\author{
John W. Dubendorff, Elena Lymar*, Frederic R. Furuya and James F. Hainfeld
}

Nanoprobes, Inc., 95 Horseblock Road Unit 1, Yaphank, New York 11980

*Biology Department, Brookhaven National Laboatory, Upton, NY 11973

We are developing a portfolio of gold nanoparticle-protein fusion tag pairs that allow specific labeling of individual protein fusion targets. This specificity of gold nanoparticle-fusion tag couples provides a means of labeling individual proteins in multisubunit, macromolecular complexes or structures with gold particles of different sizes. These pairs include a fusion tag selected by phage display (GBP: gold-binding peptide) to bind directly to $1.4 \mathrm{~nm}$ Nanogold, as well as a $5 \mathrm{~nm}$ Ni-NTA-large gold (LG) to label His-tagged targets. We are using the T7Select phage display system (Novagen) as a vehicle for testing of fusion tags.

T7 virus particles are of a size $(\sim 50 \mathrm{~nm})$ and shape as to be easily recognized in the EM. Initial experiments consisted of mixing either un-tagged or His-tagged T7 virus particles with Ni-NTA-LG (Figure 1). Preliminary results show labeling of T7 bacteriophage particles containing His-tagged capsid protein by Ni-NTA-LG. Spectral analysis showed the presence of gold in the virus peak after purification away from unbound gold by size exclusion chromatography. Untagged virions in a control separation after gold labeling showed lower absorbance at wavelengths characteristic of gold.

Examination in the EM of virions from this separation showed some T7 viruses with associated gold particles (Figure 2). Few gold labels are seen because the virus used contains predominantly wild-type capsid subunits without the His tag. An alternatve T7 cloning vector is being investigated to obtain higher numbers of gold labels on each His-tagged T7 virus particle. The use of GBP and several other fusion tags and their corresponding gold labels will be discussed. Such a collection of fusion tags and their cognate gold nanoparticles will be useful for labeling multiple protein subunits within macromolecular complexes for high resolution structural studies [1].

\section{References}

1. This research was supported by R01 GM085802 from the National Institute of General Medical Sciences. Thanks to Dr. Fred Furuya for generating EM images. 

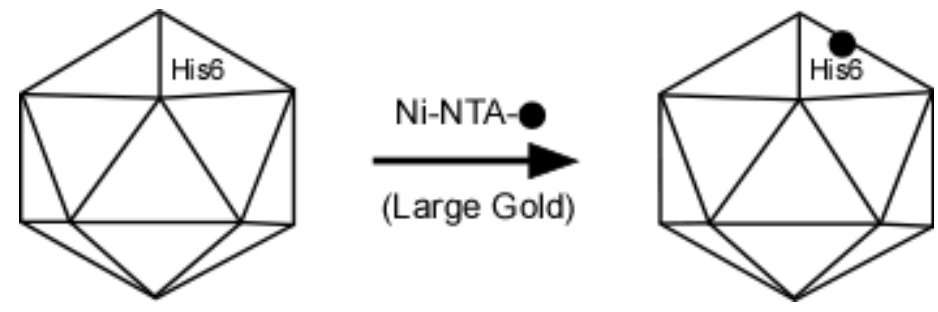

Figure 1. Labeling of His-tagged capsid protein in T7 virus particles with Ni-NTA-large gold $(5 \mathrm{~nm})$. His 6 denotes the $6 \mathrm{x}$ His tag fused to some of the capsid proteins.
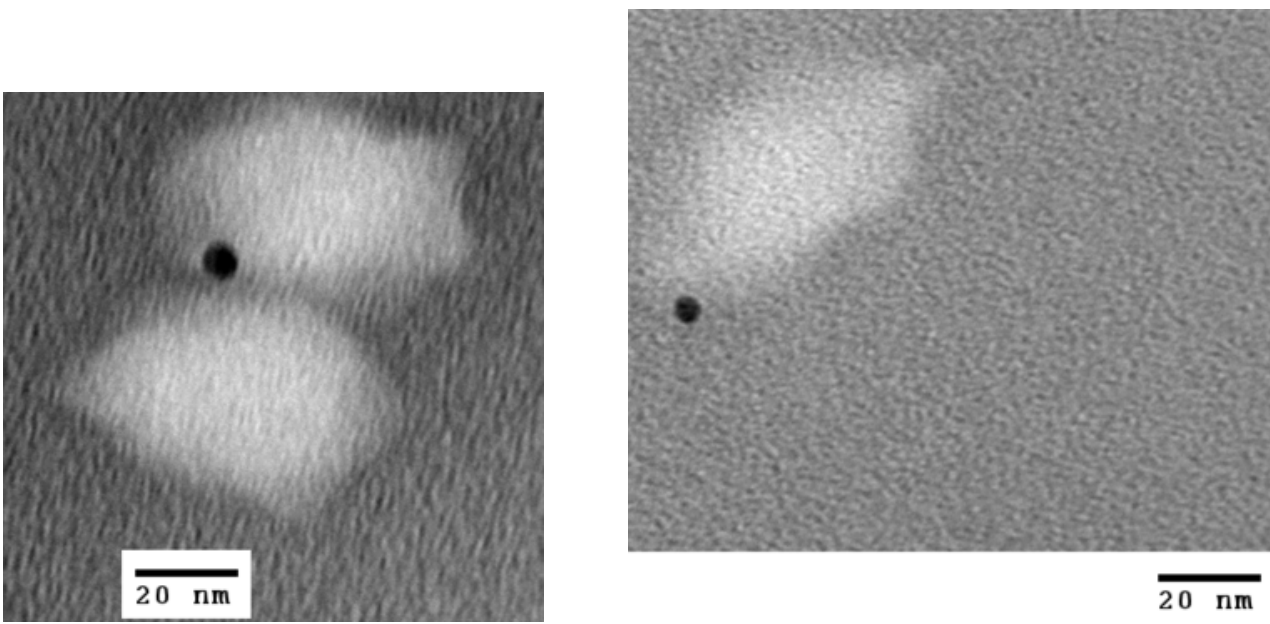

Figure 2. Negatively stained EM images of T7 virus particles labeled with $5 \mathrm{~nm}$ Ni-NTAlarge gold. 